Jpn. J. Infect. Dis., 73, 235-241, 2020

Original Article

\title{
Prediction of Contact Residues in Anti-HIV Neutralizing Antibody by Deep Learning
}

\author{
Yu Kaku ${ }^{1}$, Takeo Kuwata ${ }^{1 *}$, Miroslaw K. Gorny ${ }^{2}$, and Shuzo Matsushita ${ }^{1}$ \\ ${ }^{I}$ Clinical Retrovirology, Joint Research Center for Human Retrovirus Infection, Kumamoto University, \\ Kumamoto, Japan; and ${ }^{2}$ Department of Pathology, New York University School of Medicine, \\ New York, USA
}

\begin{abstract}
SUMMARY: The monoclonal antibody $1 \mathrm{C} 10$ targets the V3 loop of HIV-1 and neutralizes a broad range of clade $\mathrm{B}$ viruses. However, the mode of interaction between $1 \mathrm{C} 10$ and the $\mathrm{V} 3$ loop remains unclear because crystallization of $1 \mathrm{C} 10$ and the $\mathrm{V} 3$ peptide was unsuccessful due to the flexible regions present in both 1C10 and the V3 peptide. In this study, we predicted the $1 \mathrm{C} 10$ amino acid residues that make contact with the V3 loop using a deep learning (DL)-based method. Inputs from ROSIE for docking simulation and FastContact, Naccess, and PDBtools, to approximate interactions were processed by Chainer for DL, and outputs were obtained as probabilities of contact residues. Using this DL algorithm, D95, D97, P100a, and D100b of CDRH3; D53, and D56 of CDRH2; and D61 of FR3 were highly ranked as contact residues of $1 \mathrm{C} 10$. Substitution of these residues with alanine significantly decreased the affinity of $1 \mathrm{C} 10$ to the V3 peptide. Moreover, the higher the rank of the residue, the more the binding activity diminished. This study demonstrates that the prediction of contact residues using a DL-based approach is a precise and useful tool for the analysis of antibody-antigen interactions.
\end{abstract}

\section{INTRODUCTION}

The neutralizing antibody ( $\mathrm{AAb})$ against human immunodeficiency virus type 1 (HIV-1) is key to controlling HIV-1 infection (1-3). The V3 loop of HIV1 is a target for nAbs. Anti-V3 nAbs can be classified into 2 groups; ladle and cradle types, which differ based on their mode of binding to V3 peptides $(1,2)$. Ladle nAbs, represented by 447-52D, require the conserved sequence, GPGR/Q, at the crown of the V3 loop for contact. On the other hand, cradle nAbs, which possess unique features including the usage of the same immunoglobulin heavy chain (HC) gene (IGHV5-51), have V3 crown-independent binding (4-6). The anti-V3 $\mathrm{nAb}, 1 \mathrm{C} 10$, was isolated from an elite controller who maintained their viral load under detectable levels without anti-retrovirus therapy for more than 20 years (7). Although $1 \mathrm{C} 10$ is more potent against most clade B viruses, including Tier 2 viruses, compared with other anti-V3 nAbs, the mechanism of broad neutralization by $1 \mathrm{C} 10$ remains unclear (7).

Elucidating the antibody-antigen structure is essential to improving our understanding of the interaction

Received December 19, 2019. Accepted December 26, 2019. J-STAGE Advance Publication January 31, 2020.

DOI: 10.7883/yoken.JJID.2019.496

*Corresponding author: Mailing address: Clinical Retrovirology, Joint Research Center for Human Retrovirus Infection, Kumamoto University, 2-2-1 Honjo, Chuo-ku, Kumamoto 860-0811, Japan. Tel: +81 96-373-6536, Fax: +81 96-373-6537, E-mail: tkuwata@kumamoto-u.ac.jp between an antibody and an antigen. However, some proteins (e.g. membrane proteins) are difficult to crystalize while bound to their antigens (8). Flexibility is the major obstacle in crystalizing antibodyantigen complexes. In our previous study, KD-247, a humanized version of a mouse monoclonal $\mathrm{nAb}$, which targets the V3 loop $(3,9)$, was successfully crystalized. Unfortunately, we were unable to generate a crystal structure of the KD-247-V3 peptide complex (10). Crystallization of $1 \mathrm{C} 10$ and the V3 peptide was also unsuccessful, and the mode of binding involved in broad neutralization still remains unclear. Docking simulation is a useful alternative to analyze antibody-antigen interactions. However, the prediction of contact residues by this method is not always considered and is elusive in docking simulation (11-13). In addition, although simulation does not always require a crystal structure, the success rate of the docking is highly dependent on the source of the complex components to be simulated, especially backbones which have been constructed by homology modeling $(14,15)$.

Deep learning (DL) has been applied to biological research and accumulating evidence has proven its prediction capability for elucidating the binding mode of anti-HIV nAbs (16-18). In the present study, we predicted the contact residues of anti-V3 nAb $1 \mathrm{C} 10$ using the DL-based method to reveal residues that are important for binding to the V3 loop.

\section{MATERIALS AND METHODS}

Docking simulation of crystal structure and homology models by Rosetta Online Server that 


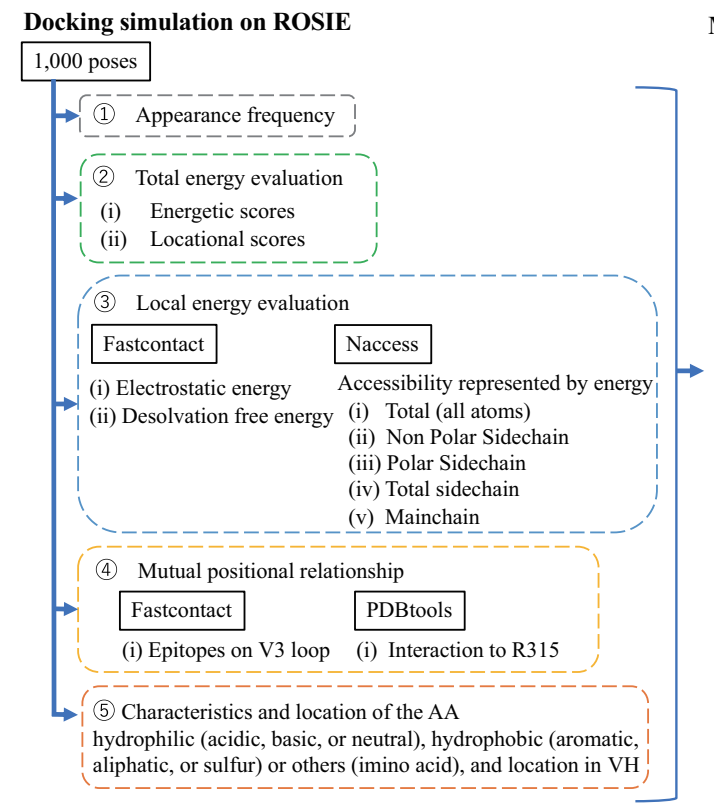

\section{Multilayer perceptron (MLP)}

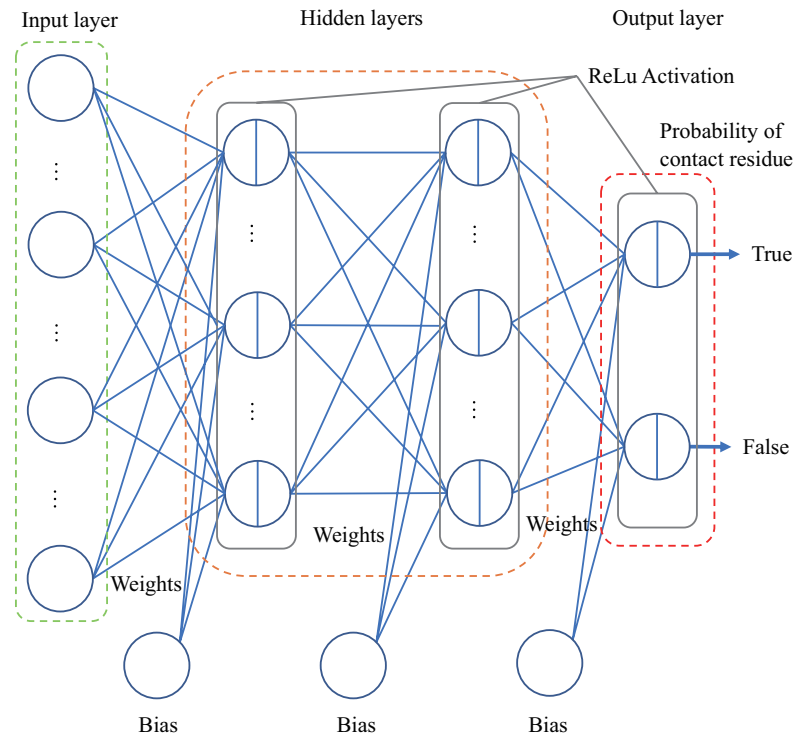

Fig. 1. (Color Online) Approach for the prediction of contact residues in anti-V3 nAb. Docking simulation was performed by ROSIE, and 1,000 docked poses were generated. Local energy and AA positions were calculated by FastContact, Naccess, and PDBtools. Parameters from these analyses were normalized and used as inputs for DL on Chainer. Multi-layer perceptron (MLP) was constructed by an input layer including 43 nodes, 2 hidden layers including 10 nodes per layer, and 1 output layer including 2 nodes. The output of this MLP was either "true contact residue" or "false contact residue."

Includes Everyone (ROSIE): ROSIE contains a proteinprotein docking simulation based on RosettaDock, which is freely available at (https://rosie.graylab.jhu. edu/ligand_docking) as reported by Lykcov et al. (19). We chose the Docking2 protocol on ROSIE to output 1,000 decoys each using the crystal structure of anti-V3 nAbs including 447-52D (Protein Data Bank [PDB] ID: 4M1D) (20), 1334 (PDB ID: 6DB7) (5), F425 B4e8 (PDB ID: 2QSC) (21), and 311-11D (PDB ID: 6DB6) (5), of which, the HC, light chain (LC), and V3 loop were separately used as templates for the homology modeling of 1C10 by Swiss-model (22) followed by combining an alignment with the original crystal structure on Pymol (23). The crystal structures and the homology models were uploaded to the ROSIE server to generate a docking score (24-29).

Estimation of the credibility of contact residues in docking poses: To identify the amino acids (AAs) involved in the interaction in the 1,000 docked poses from ROSIE, additional analyses were performed. The interaction between the $\mathrm{HC}$ and the V3 loop was analyzed using FastContact (30) and Naccess (31). Residues in the range of $5 \AA$ from Arg315 of the V3 loop were independently determined by contact.py in PDBtools (32). The unbound antibody structure was extracted using BioPython (33). Character and structural positions on the V3 loop (34) or on the variable region of the $\mathrm{HC}$ were also used as parameters. Comparison of these parameters was performed using the mean and $P$ values of the data, which were calculated using the Welch's $t$-test of each value obtained by Python (35). Boxplots are generated in Matplotlib (36).

Deep learning by Chainer: To standardize the input data, input parameters were converted to a range from 0 to 1 for continuous variants and 0 or 1 for objective variants. Blank data were filled by the mean value of the 1,000 poses in respective categories. A multi-layer perceptron (MLP) was composed by 1 input layer including 43 nodes, 2 hidden layers including 10 nodes per layer, and 1 output layer including 2 nodes (Fig. 1). DL was performed on Chainer (37) using rectified linear unit as an activation function, batch size $=5$, and epoch $=20$. The results obtained from 3,000 simulations using the crystal structures of 1334, F425 B4e8, and 311-11D were randomly separated to a teacher model $(30 \%)$ and a student model (70\%). After training with the teacher model, the model was applied to the output of ROSIE simulating the 1C10 homologous model to 1334. Relative frequency selected as a contact residue in the prediction was calculated by the number selected as a contact residue at each residue divided by the total number selected as a contact residue at all the residues.

Analysis of binding activity of $1 \mathrm{C} 10$ mutants to the V3 peptide: The 7 predicted contact residues of $1 \mathrm{C} 10$ were substituted with an alanine by PCR mutagenesis $(7,36)$. The resultant $1 \mathrm{C} 10 \mathrm{HC}$ mutants, 1C10drCDR2-m (D53A, D56A, and D61A), 1C10drCDR3-m (D95A, D97A, D100aA, and P100bA), dr95-m (D95A), dr97-m (D97A), dr100a-m (D100aA), and dr100b-m (P100bA) were transfected into HEK293 cells with a 1C10 LC expressing vector using the FectoPro Expression System (PolyPlus, Illkirch, France). The binding activity of the culture supernatants of transfected cells was analyzed by enzyme-linked immunosorbent assay (ELISA) to the V3 peptide of JRFL (NNT20; NNTRKSIHIGPGRAFYTTGE), as previously described (7). The AA positions in 1C10 are shown according to the Kabat numbering scheme.

\section{RESULTS}

$1 \mathrm{C} 10$ homology modeling using anti-V3 
nAb Contact Residue Prediction by Deep Learning

\begin{tabular}{|c|c|c|c|c|}
\hline & 1334 & F425 B4e8 & 311-11D & 447-52D \\
\hline \multicolumn{5}{|c|}{ Docking simulation of $1 \mathrm{C} 10$ homology model } \\
\hline ROSIE docking score & -195.30 & -186.58 & -121.67 & -35.887 \\
\hline \multicolumn{5}{|l|}{ Original crystal structure } \\
\hline ROSIE docking score & -221.41 & -227.70 & -223.47 & -223.84 \\
\hline Contact residues on $\mathrm{HC}$ & $\begin{array}{l}\text { W50, N52 G52a, D53, } \\
\text { T57, Y97, D99, P100i }\end{array}$ & N100d & $\begin{array}{l}\text { T31, R50, N52, G97, D98, } \\
\text { Y100, I100c, W100i }\end{array}$ & $\begin{array}{l}\text { W33, D95, D100f, Y100g, } \\
\text { Y100h, Y100j }\end{array}$ \\
\hline CDRH3 RMSD $(\AA)$ & - & 3.43 & 3.38 & 1.45 \\
\hline CDRH3 length & 22 & 14 & 14 & 20 \\
\hline V3 loop RMSD $(\AA)$ & - & 2.25 & 0.68 & 3.69 \\
\hline V3 sequence & RKRIHIGPGRAFY & RKRIHIGPGRAFYTT & RKRIHIGPGRAFYT & CRKRIHIGPGRAFYTC \\
\hline
\end{tabular}

Fig. 2. Comparison of docking scores of cognate and homology models among 4 anti-V3 antibody crystal structures. The best docking scores obtained from ROSIE docking simulation of 1C10 models based on 1334, F425 B4e8, 311-11D, and 44752D structures are shown. The redocking simulation of 4 anti-V3 antibodies was also performed using their intact structures on PDB. Root mean square distance (RMSD) of the top 10 output complexes was calculated by PyMol.

antibody-V3 structures: Homology models of anti-V3 $\mathrm{nAb} 1 \mathrm{C} 10$ were analyzed by docking simulation using ROSIE based on the structures of the anti-V3 antibodies, 447-52D, 1334, F425 B4e8, and 311-11D. Thousands of poses were generated through the lowresolution stage followed by the high-resolution stage, and 1,000 poses were selected from the lowest total energy score. ROSIE also calculated values categorized as energy fractions including fa_atr, fa_dun, fa_elec, fa pair, fa rep, fa sol, 1 sc, and total score, and the location of a pose containing rms, 1rms, cen_rms, and Fnat.

The 1C10 homology model with a typical ladle-type antibody, 447-52D, resulted in a significantly higher docking score $(-35.887)$ compared to the 447-52D crystal structure (Fig. 2). The 1C10 homology model using 311-11D showed a moderate score $(-121.67)$. The models using 1334 and F425 B4e8 showed low docking scores $(-195.30$ and -186.58 , respectively) which were close to the scores of the original crystal structures. The binding mode of $\mathrm{F} 425 \mathrm{~B} 4 \mathrm{e} 8$ is unique and uses only $1 \mathrm{AA}$ on the HC to make contact with the V3 loop, because the LC harbors the main points of contact with the V3 loop. However, 1334 and 311-11D used closelyrelated AAs of complementarity-determining region 3 of heavy chain (CDRH3) for binding to the V3 loop (Fig. 2). Therefore, we selected 3 binding models with the lowest docking scores using 1334, F425 B4e8, and $311-11 \mathrm{D}$, but excluded the model that used 447$52 \mathrm{D}$, for further analysis. We focused on the interaction with HC for 2 reasons. Firstly because the dominance of CDRH3 in the anti-V3 antibody-V3 contacts, as is commonly observed for antigen-recognition by general immunoglobulin. Secondly, because this allows for faster calculation for the detection of contact residues in the analyses.

Analysis of contact residues in docking poses: Of the residues identified as contact residues from existing crystal structures, N100d in F425 B4e8 was the top-ranked contact residue by homology modeling. However, the top-ranked contact residues in the 1334 and 311-11D models were not the contact residues in the crystal structure (Fig. 2 and Table 1). The actual binders identified by the crystal structure were not always as highly ranked as the contact residues in the redocking poses from ROSIE, although they were listed in the top 20 ranks (Table 1 ).

To obtain data for contact residues, each AA residue from the 1,000 poses generated by ROSIE was analyzed by Fastcontact, Naccess, and PDBtools (Fig. 1). FastContact ranked the interaction between residues on the $\mathrm{HC}$ and V3 peptide by electrostatic force and free energy and provided energetic values containing the epitope positions on the V3 loop involved in the HCrelated interactions. Naccess analyzed the type and/ or polarity of AA branches engaged in the interaction by comparing the solvent-accessible surface area of a receptor to a receptor-ligand complex. PDBtools was used as a pickup tool for uncommon attractive forces such as salt bridge or cation- $\pi$ interaction with a distance of $5 \AA$ from R315 on the V3 loop, rather than hydrogen bonds or van der Waals forces. The parameters used to predict contact residues included: (i) appearance frequency as a contact residue in the 1,000 simulated poses from ROSIE; (ii) total energy evaluation in ROSIE output including energetic and locational scores; (iii) energy at the binding sites including electrostatic force and free energy calculated by Fastcontact and interaction energy at AAs on the accessible surface determined by Naccess; and (iv) interaction with epitopes on the V3 loop by Fastcontact, especially with Arg315 which is critical for typical anti-V3 abs-V3 loop interactions, using PDBtools.

Prediction of contact residues of $1 \mathrm{C} 10$ by MLP neural network algorithm: MLP was developed to predict contact residues in anti-V3 antibody-V3 peptide complexes by DL (Fig. 2). To avoid overfitting to the 1334 model, we used parameters obtained from 1,000 docking poses of each of the 3 homology models using 1334, F425 B4e8, and 311-11D. In addition to using output values from ROSIE, FastContact, Naccess, and PDBtools for DL, we used AA characteristics, such as polarity, charge, hydrophobicity, and location of the immunoglobulin variable region. Thirty percent of data sets were used as a teacher model to train the DL algorithm by Chainer, and the remaining $70 \%$ was 
Table 1. Appearance frequency ${ }^{1)}$ as contact residues in docking poses

\begin{tabular}{|c|c|c|c|c|c|c|c|c|c|c|c|c|}
\hline \multirow[b]{2}{*}{ Rank } & \multicolumn{4}{|c|}{1334} & \multicolumn{4}{|c|}{$\mathrm{F} 425 \mathrm{~B} 4 \mathrm{e} 8$} & \multicolumn{4}{|c|}{ 311-11D } \\
\hline & $\mathrm{AA}$ & freq & actual & pred & AA & freq & actual & pred & AA & freq & actual & pred \\
\hline 1 & N100f & 18.36 & - & 18.12 & N100d & 13.89 & + & 99.86 & Y99 & 17.78 & - & 0.00 \\
\hline 2 & D100e & 13.84 & - & 0.74 & D95 & 9.44 & - & 0.00 & D98 & 12.92 & + & 38.89 \\
\hline 3 & N100d & 13.52 & - & 0.00 & N61 & 8.40 & - & 0.00 & E100b & 9.18 & - & 0.00 \\
\hline 4 & P100g & 9.95 & - & 0.00 & D53 & 6.93 & - & 0.00 & E95 & 6.99 & - & 0.00 \\
\hline 5 & T100b & 7.36 & - & 0.00 & T57 & 6.32 & - & 0.00 & N52 & 6.78 & + & 20.73 \\
\hline 6 & D99 & 5.81 & + & 42.03 & E55 & 6.22 & - & 0.00 & Y100 & 6.51 & + & 19.91 \\
\hline 7 & G100c & 4.11 & - & 0.00 & T56 & 6.20 & - & 0.00 & S100a & 6.27 & - & 1.12 \\
\hline 8 & E58 & 4.08 & - & 0.00 & E99 & 5.29 & - & 0.00 & N54 & 5.65 & - & 2.53 \\
\hline 9 & P98 & 3.50 & - & 0.00 & G10b & 3.97 & - & 0.00 & D56 & 5.41 & - & 0.00 \\
\hline 10 & A100h & 3.18 & - & 0.00 & Y58 & 3.83 & - & 0.00 & S100d & 3.31 & - & 0.03 \\
\hline 11 & V100a & 3.12 & - & 0.00 & S52 & 3.59 & - & 0.00 & S96 & 2.36 & - & 0.01 \\
\hline 12 & D54 & 2.39 & - & 0.15 & H59 & 3.57 & - & 0.00 & T31 & 2.21 & + & 6.77 \\
\hline 13 & N52 & 1.98 & + & 14.01 & $\mathrm{~S} 100 \mathrm{c}$ & 2.67 & - & 0.00 & R58 & 2.02 & - & 0.00 \\
\hline 14 & Y97 & 1.14 & + & 8.91 & S100a & 2.42 & - & 0.00 & $\mathrm{I} 100 \mathrm{c}$ & 1.38 & + & 4.14 \\
\hline 15 & P100i & 0.96 & + & 5.30 & Y97 & 2.07 & - & 0.00 & T57 & 1.34 & - & 0.00 \\
\hline 16 & D53 & 0.83 & + & 6.29 & L64 & 1.61 & - & 0.00 & N28 & 1.32 & - & 0.00 \\
\hline 17 & T57 & 0.83 & + & 0.00 & S49 & 1.57 & - & 0.00 & G97 & 1.20 & + & 3.65 \\
\hline 18 & $\mathrm{P} 100 \mathrm{j}$ & 0.76 & - & 1.44 & Y98 & 1.39 & - & 0.00 & Y32 & 1.14 & - & 0.00 \\
\hline 19 & L56 & 0.61 & - & 0.00 & S52a & 1.25 & - & 0.00 & T30 & 1.06 & - & 0.00 \\
\hline 20 & W50 & 0.55 & + & 0.00 & N73 & 1.07 & - & 0.00 & R50 & 0.92 & + & 1.21 \\
\hline Total & & 96.88 & & 96.99 & & 91.70 & & 99.86 & & 95.75 & & 98.9 \\
\hline
\end{tabular}

${ }^{1)}$ : Relative frequencies (\%) identified as a contact residue (freq) in the ROSIE-docking poses and a predicted contact residue by DL (pred) are shown. These frequencies were calculated by the number selected as a contact residue at each residue divided by a total number selected as a contact residue at all the residues. Contact residues in the crystal structure (actual) are shown as (+).

used as a student model to check the accuracy of the algorithm for the untrained model as well as to avoid overfitting to the teacher model. During training on the teacher model, the accuracy reached more than $95 \%$ for the teacher and the student model (Fig. 3). The MLP algorithm successfully identified contact residues in models 1334, F425 B4e8, and 311-11D, irrespective of their appearance rate in the docking simulation (Table 1). Most actual contact residues in the crystal structures were frequently selected as contact residues in the prediction of all 3 models by DL, although T57 and W50 in 1334 were not predicted as contact residues. In contrast, the residues that were highly ranked in the docking simulation, but were not actual binders, were rarely selected as contact residues by DL.

This MLP algorithm was applied to predict the $1 \mathrm{C} 10$ contact residues. DL was performed using 1,000 docking poses of the 1C10 homology model, which was based on the 1334-V3 structure, because of the lowest docking score among anti-V3 antibodies (Fig. 2 ). The AA residues predicted as contact residues were mainly in the CDRH3 and CDRH2 (Fig. 4A and Table 2 ). The top 10-ranked AA residues were 5 residues in the $\mathrm{CDRH} 3,3$ residues in the $\mathrm{CDRH} 2,1$ residue in the CDRH1, and 1 residue in FR3.

Importance of the predicted $A A$ residues on $1 \mathrm{C10}$ binding activity: To verify the contact residues

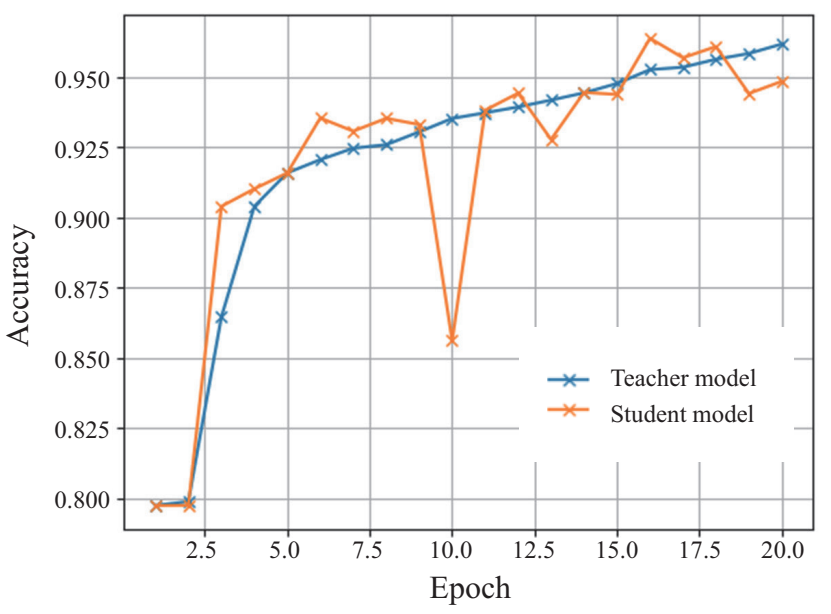

Fig. 3. (Color Online) Learning to improve accuracy on deeplearning models. During 20 epochs containing a forward and a backward pass per an epoch, the accuracy on the $30 \%$ of the total data as a teacher model reached at more than $97 \%$ and on the $70 \%$ of the total data as a student model reached at approximately $95 \%$.

predicted by this DL-based method, 1C10 mutants with substitutions in the predicted residues were constructed, and their affinity for the V3 peptide was studied. The mutant 1C10drCDR3-m, in which high-ranked residues 
A

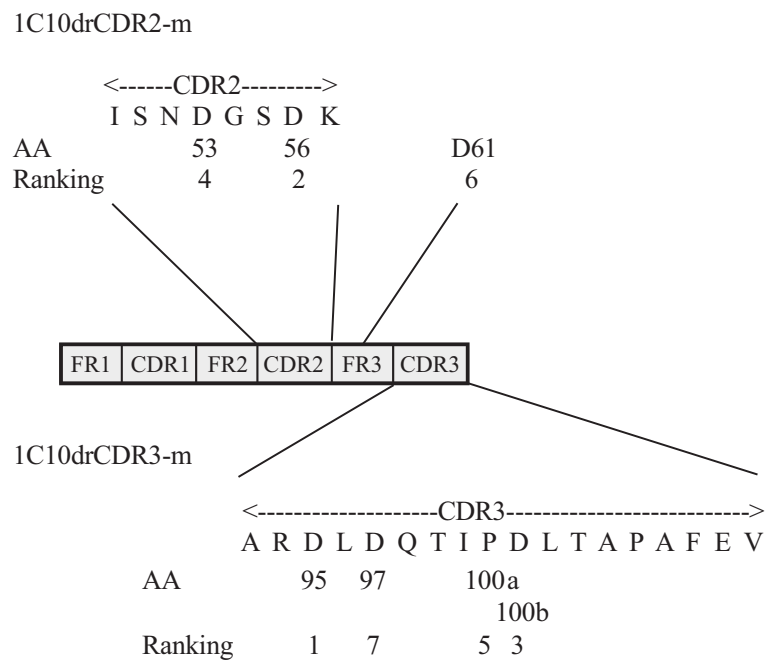

B

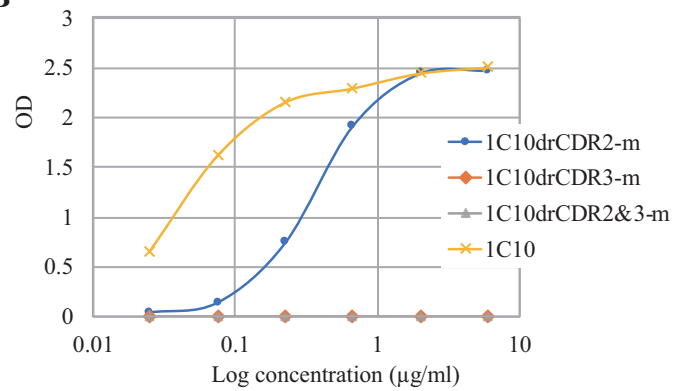

C

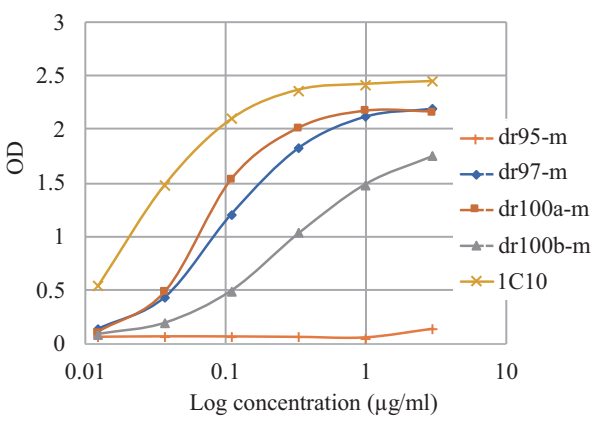

Fig. 4. (Color Online: $\mathrm{B}$ and $\mathrm{C}$ ) Binding affinity of $1 \mathrm{C} 10$ mutants to the $\mathrm{V} 3$ peptide. Effect of alanine substitution at AA residues predicted in the top 7 contact residues of $1 \mathrm{C} 10 \mathrm{HC}$ by DL was examined by ELISA using the V3 peptide, JRFL NNT20. (A) The location of the top 7 AA residues in 1C10 are shown schematically. The AA residues in CDRH2 and FR3 were substituted to alanine in the $1 \mathrm{C} 10$ mutant, 1C10drCDR2-m. The AA residues in CDRH3 were substituted to alanine in the mutant, 1C10drCDR3-m. The mutant, 1C10drCDR2\&3-m, contained all 7 substitutions. Point mutants with D95A, D97A, P100aA, and D100bA were also constructed, and designated as dr95-m, dr97-m, dr100a-m, and dr100b-m, respectively. (B) Binding activities of 1C10 mutants, 1C10drCDR2-m, 1C10drCDR3-m, and 1C10drCDR2\&3-m, were compared. (C) Binding activities of 1C10 point mutants, dr95-m, dr97-m, dr100a-m, and dr100b-m, were compared.

in the CDRH3, D95, D97, P100a, and D100b, were mutated to an alanine, did not bind to the V3 peptide at all (Fig. 4A and B). The mutant 1C10drCDR2-m, in which D53 and D56 in the CDRH2 and D61 in FR3 were mutated to an alanine, showed significantly reduced binding to the $\mathrm{V} 3$ peptide. The complete loss of $1 \mathrm{C} 10$ binding activity by CDRH3 mutations was further analyzed using point mutants (Fig. 4C). The substitution D95 to an alanine, which was the most frequently predicted contact residue (Fig. 4A and Table 2), abolished $1 \mathrm{C} 10$ binding activity. Substitution at $\mathrm{D} 100 \mathrm{~b}$, the third ranked residue markedly decreased $1 \mathrm{C} 10$ binding activity. Substitution at P100a, the fifth ranked residue, and $\mathrm{D} 97$, the seventh ranked residue, moderately decreased binding activity. These results suggest that the contact residues predicted by the DLbased method are important for $1 \mathrm{C} 10-\mathrm{V} 3$ binding, and that ranking of residues by DL-based prediction is linked to their contribution to binding.

\section{DISCUSSION}

We have developed an MLP algorithm to predict the contact residues in an anti-V3 antibody-V3 complex in silico. Furthermore, we have shown that this DLbased method can precisely predict residues which are important for in vitro binding. The precise prediction of contact residues demonstrates the usefulness of the DL-based method for understand antibody-antigen interactions, especially for complexes that are difficult to crystalize. Moreover, this study suggests that the prediction of contact residues using this DL-based method with various parameters from multiple analyses is much better than using a simple docking simulation. Importantly, the biological data, which were obtained by neutralization assay of mutant antibodies, support our computational analyses. For the first time, we demonstrate that DL can be used to predict which AA residues are involved in the interaction between the anti-V3 antibody and the V3 loop of HIV-1.

Although homologous modeling using the ladle-type antibody, 447-52D, was not readily adaptable to $1 \mathrm{C} 10$ (Fig. 2) $(1,20)$, homology modeling of $1 \mathrm{C} 10$ using other types of anti-V3 antibodies, 1334, F425 B4e8, and 311$11 \mathrm{D}$, was preferable. Two antibodies, 1334 and 311$11 \mathrm{D}$, showed binding to the V3 loop mainly by HC, whereas F425 B4e8 bound mainly to the LC, which was in contrast to the HC-dependent binding exhibited by other anti-V3 antibodies. We selected 1334, F425 B4e8, and $311-11 \mathrm{D}$ for $1 \mathrm{C} 10$ homology modeling by ROSIE docking scores instead of binding modes. Although contact residues were variable among these 3 antibodies in crystal structures, the contact residues predicted by the DL-based method were consistent with the actual contact residues (Table 1).

Most binding residues identified in crystal structure analysis were ranked in the top 20 frequent binders in the docking simulation by ROSIE (Table 1). However, the rank of binders in the simulation did not correspond to the binders in the crystal structure. Although N100d of F425 B4e8, which is the only actual contact residue in the $\mathrm{HC}$ of this antibody, was most frequently identified as a contact residue, the rankings of the actual contact residues in 1334 and 311-11D were not high 
Table 2. Contact residues of $1 \mathrm{C} 10$ predicted by DL-based method

\begin{tabular}{|c|c|c|c|c|}
\hline Region & $\mathrm{HC}^{1)}$ position & HC AA & predict $^{2)}$ & ranking ${ }^{3)}$ \\
\hline \multirow[t]{9}{*}{ CDR3 } & 95 & ASP & 26.23 & 1 \\
\hline & $100 \mathrm{~b}$ & ASP & 17.37 & 3 \\
\hline & $100 \mathrm{a}$ & PRO & 6.42 & 5 \\
\hline & 97 & ASP & 4.94 & 7 \\
\hline & $100 \mathrm{f}$ & PRO & 2.4 & 8 \\
\hline & 100 & ILE & 0.35 & 11 \\
\hline & 104 & GLU & 0.22 & 14 \\
\hline & $100 \mathrm{e}$ & ALA & 0.09 & 17 \\
\hline & $100 \mathrm{c}$ & LEU & 0.09 & 17 \\
\hline \multirow[t]{7}{*}{ CDR2 } & 56 & ASP & 23.63 & 2 \\
\hline & 53 & ASP & 9.14 & 4 \\
\hline & $52 \mathrm{a}$ & ASN & 0.85 & 10 \\
\hline & 54 & GLY & 0.28 & 12 \\
\hline & 50 & ILE & 0.22 & 14 \\
\hline & 51 & ILE & 0.03 & 19 \\
\hline & 52 & SER & 0.03 & 19 \\
\hline \multirow[t]{3}{*}{ CDR1 } & 31 & ASN & 1.93 & 9 \\
\hline & 28 & MET & 0.06 & 19 \\
\hline & 33 & ALA & 0.03 & 19 \\
\hline \multirow[t]{4}{*}{ FR3 } & 61 & ASP & 5.22 & 6 \\
\hline & 58 & TYR & 0.25 & 13 \\
\hline & 65 & GLN & 0.13 & 16 \\
\hline & 75 & ASN & 0.03 & 19 \\
\hline FR2 & 4 & GLU & 0.03 & 19 \\
\hline
\end{tabular}

in the redocking simulation. This is partially due to the calculation of energy by ROSIE, which does not consider the component amino acids involved in the interaction (24-27). Analysis by Fastcontact, Naccess, and PDBtools contributed to the precise prediction of contact residues by the addition of local energy and locational parameters (30-32). DL is suitable for the comprehensive analysis of various parameters obtained from multiple analyses (16-18). The precise prediction of contact residues in this study demonstrates the usefulness of the DL-based method using various parameters from multiple analyses to reveal antibodyantigen interactions.

In summary, with supporting data of local energy on the contact sites, our DL algorithm predicted the contact residues of the anti-V3 nAb, 1C10, for the V3 loop. The contact residues predicted by the DL-based method were shown to be important for binding to the V3 loop. We focused on the identification of contact residues in the paratope of $1 \mathrm{C} 10$ in this study. Prediction of contact residues in the epitope in the V3 loop using this DL algorithm will be useful to further elucidate the interaction between anti-V3 nAbs and the V3 loop. Ascertaining both the epitope and paratope contact residues will lead help us understand the mechanism by which $1 \mathrm{C} 10$ is able to neutralize a broad range of clade $\mathrm{B}$ viruses.

Acknowledgments We thank Miki Tsukiashi for her kind administrative assistance. This work was supported by JSPS KAKENHI (JP18H02854) and AMED (JP19fk0410025h0001).

Conflict of interest None to declare.

\section{REFERENCES}

1. Balasubramanian P, Kumar R, Williams C, et al. Differential induction of anti-V3 crown antibodies with cradle- and ladlebinding modes in response to HIV-1 envelope vaccination. Vaccine. 2017; 35: 1464-73.

2. Hessell AJ, McBurney S, Pandey S, et al. Induction of neutralizing antibodies in rhesus macaques using V3 mimotope peptides. Vaccine. 2016; 34: 2713-21.

3. Matsushita S, Yoshimura K, Ramirez KP, et al. Passive transfer of neutralizing $\mathrm{mAb} \mathrm{KD}-247$ reduces plasma viral load in patients chronically infected with HIV-1. AIDS. 2015; 29: 453-62.

4. Gorny MK, Sampson J, Li H, et al. Human anti-V3 HIV1 monoclonal antibodies encoded by the VH5-51/VL lambda genes define a conserved antigenic structure. PLoS One. 2011; 6: e27780. 
5. Chan KW, Pan R, Costa M, et al. Structural comparison of human anti-HIV-1 gp120 V3 monoclonal antibodies of the same gene usage induced by vaccination and chronic infection. J Virol. 2018; 92. pii: e00641-18.

6. Gorny MK, Wang XH, Williams C, et al. Preferential use of the VH5-51 gene segment by the human immune response to code for antibodies against the V3 domain of HIV-1. Mol Immunol. 2009; 46: 917-26.

7. Ramirez Valdez KP, Kuwata T, Maruta Y, et al. Complementary and synergistic activities of anti-V3, CD4bs and CD4i antibodies derived from a single individual can cover a wide range of HIV-1 strains. Virology. 2015; 475: 187-203.

8. Carpenter EP, Beis K, Cameron AD, et al. Overcoming the challenges of membrane protein crystallography. Curr Opin Struct Biol. 2008; 18: 581-6.

9. Eda Y, Takizawa M, Murakami T, et al. Sequential immunization with V3 peptides from primary human immunodeficiency virus type 1 produces cross-neutralizing antibodies against primary isolates with a matching narrowneutralization sequence motif. J Virol. 2006; 80: 5552-62.

10. Kirby KA, Ong YT, Hachiya A, et al. Structural basis of cladespecific HIV-1 neutralization by humanized anti-V3 monoclonal antibody KD-247. FASEB J. 2015; 29: 70-80.

11. Englebienne P, Fiaux H, Kuntz DA, et al. Evaluation of docking programs for predicting binding of Golgi alpha-mannosidase II inhibitors: a comparison with crystallography. Proteins. 2007; 69: 160-76.

12. Bohari MH, Sastry GN. FDA approved drugs complexed to their targets: evaluating pose prediction accuracy of docking protocols. J Mol Model. 2012; 18: 4263-74.

13. Pierce BG, Wiehe K, Hwang H, et al. ZDOCK server: interactive docking prediction of protein-protein complexes and symmetric multimers. Bioinformatics. 2014; 30: 1771-3.

14. Shih ESC, Hwang MJ. A critical assessment of informationguided protein-protein docking predictions. Mol Cell Proteomics. 2013; 12: 679-86.

15. Kilambi KP, Gray JJ. Structure-based cross-docking analysis of antibody-antigen interactions. Sci Rep. 2017; 7: 8145.

16. Wójcikowski M, Ballester PJ, Siedlecki P. Performance of machine-learning scoring functions in structure-based virtual screening. Sci Rep. 2017; 7: 46710.

17. Conti S, Karplus M. Estimation of the breadth of CD4bs targeting HIV antibodies by molecular modeling and machine learning. PLoS Comput Biol. 2019; 15: e1006954.

18. Bricault CA, Yusim K, Seaman MS, et al. HIV-1 neutralizing antibody signatures and application to epitope-targeted vaccine design. Cell Host Microbe. 2019; 25: 59-72. e8.

19. Lyskov S, Chou FC, Conchúir SÓ, et al. Serverification of molecular modeling applications: the rosetta online server that includes everyone (ROSIE). PLoS One. 2013; 8: e63906.

20. Killikelly A, Zhang HT, Spurrier B, et al. Thermodynamic signatures of the antigen binding site of mAb 447-52D targeting the third variable region of HIV-1 gp120. Biochemistry. 2013; 52: 6249-57.
21. Bell CH, Pantophlet R, Schiefner A, et al. Structure of antibody F425-B4e8 in complex with a V3 peptide reveals a new binding mode for HIV-1 neutralization. J Mol Biol. 2008; 375: 969-78.

22. Waterhouse A, Bertoni M, Bienert S, et al. SWISS-MODEL: homology modelling of protein structures and complexes. Nucleic Acids Res. 2018; 46: W296-W303.

23. DeLano WL. PyMOL 2.3. San Carlos, CA: DeLano Scientific; 2002. Available at $<$ http://www.pymol.org/>. Accessed October $31,2019$.

24. Chaudhury S, Berrondo M, Weitzner BD, et al. Benchmarking and analysis of protein docking performance in Rosetta v3.2. PLoS One. 2011; 6: e22477.

25. Gray JJ, Moughon S, Wang C, et al. Protein-protein docking with simultaneous optimization of rigid-body displacement and sidechain conformations. J Mol Biol. 2003; 331: 281-99.

26. O’Meara MJ, Leaver-Fay A, Tyka MD, et al. Combined covalentelectrostatic model of hydrogen bonding improves structure prediction with Rosetta. J Chem Theory Comput. 2015; 11: 60922.

27. Alford RF, Leaver-Fay A, Jeliazkov JR, et al. The rosetta allatom energy function for macromolecular modeling and design. J Chem Theory Comput. 2017; 13: 3031-48.

28. Lensink MF, Wodak SJ. Docking and scoring protein interactions: CAPRI 2009. Proteins. 2010; 78: 3073-84.

29. Basu S, Wallner B. Finding correct protein-protein docking models using ProQDock. Bioinformatics. 2016; 32: i262-i270.

30. Camacho CJ, Zhang C. FastContact: rapid estimate of contact and binding free energies. Bioinformatics. 2005; 21: 2534-6.

31. Hubbard SJ, Thornton JM. NACCESS, Computer Program, Department of Biochemistry and Molecular Biology, University College London. 1993. Available at <http://www.bioinf. manchester.ac.uk/naccess/>. Accessed October 31, 2019.

32. Harms M. A set of tools for manipulating and doing calculations on wwPDB macromolecule structure files. Available at $<$ https:// github.com/harmslab/pdbtools/>. Accessed October 31, 2019.

33. Cock PJA, Antao T, Chang JT, et al. Biopython: freely available python tools for computational molecular biology and bioinformatics. Bioinformatics. 2009; 25: 1422-3.

34. Jiang X, Burke V, Totrov M, et al. Conserved structural elements in the V3 crown of HIV-1 gp120. Nat Struct Mol Biol. 2010; 17: 955-61.

35. Python Software Foundation. Python Language Reference, version 2.7. Available at $<$ http://www.python.org/>. Accessed October 31, 2019.

36. JD Hunter. Matplotlib: a 2D graphics environment. Computing Science Engineering. 2007; 9: 90-5.

37. Tokui S, Oono K, Hido S, et al. Chainer: a next-generation open source framework for deep learning. Proceedings of workshop on machine learning systems (LearningSys) in the twenty-ninth annual conference on neural information processing systems (NIPS) 2015; 5. Available at <http://learningsys.org/papers/ LearningSys_2015_paper_33.pdf>. Accessed April 15, 2020. 